\title{
149. On Boundary Values of Some Pseudo-Analytic Functions
}

\author{
By Kêichi Shibata
}

(Comm. by K. KunUGI, M.J.A., Dec. 12, 1957)

Let $\zeta=\varphi(z)$ be a quasi-conformal mapping from $|z|<1$ to $|\zeta|<1$. Then $\varphi(z)$ is not necessarily absolutely continuous function of $\arg z=t$ on $|z|=1$ (cf. [4]), although it is always continuous and of bounded variation for $0 \leq t \leq 2 \pi$. In the present short note we shall give a sufficient condition, for $\varphi\left(e^{i t}\right)$ to be absolutely continuous in $t$, in such a form, that it is applicable to generalization of some classical theorems. Our analysis is based essentially on Ahlfors's mapping theory $[2,3]$. We must also remark that the result is closely related to one of the propositions stated in [5] without proof.

In this paper we use the following notations: for any complex number $z, z^{*}$ is its inversion with respect to the unit circumference. Areal mean of a continuous function $g(z)$ over the disk $|z-a| \leq b$ shall be denoted by $M(g ; a ; b)$, i.e.

$$
M(g ; a ; b)=\frac{1}{\pi b^{2}} \int_{0}^{b} \int_{0}^{2 \pi} g\left(a+r e^{i l}\right) r d t d r .
$$

Any integral without explicit indication of its integration domain should be computed over the whole plane.

Lemma. Given any function $g(z)$ in $|z|<1$ which fulfils the Hölder condition of order $\alpha$

$$
\left|g\left(z_{1}\right)-g\left(z_{2}\right)\right| \leq A\left|z_{1}-z_{2}\right|^{\alpha} \quad\left|z_{1}\right|<1,\left|z_{2}\right|<1,0<\alpha \leq 1,
$$

then there exists a sequence of functions $\left\{g_{n}(z)\right\}$ in $|z|<\infty$, such as to satisfy the conditions

i) $g_{n}(z)$ has a uniformly bounded carrier,

ii) $\left|g_{n}\left(z_{1}\right)-g_{n}\left(z_{2}\right)\right| \leq B\left|z_{1}-z_{2}\right|^{\alpha}$,

iii) $\sup _{|z|<\infty}\left|g_{n}(z)\right| \leq \sup _{|z|<1}|g(z)|$,

$$
\left(\begin{array}{l}
\left|z_{1}\right|<\infty,\left|z_{2}\right|<\infty \\
n=1,2, \cdots
\end{array}\right)
$$

iv) $\left\{g_{n}(z)\right\}$ converges uniformly to $g(z)$ in $|z|<1$.

Proof. For example we proceed as follows:

Set

$$
\gamma(z)=\left\{\begin{array}{lr}
g\left(z^{*}\right) & 1<|z| \leq 4 \\
0 & |z| \geq 5 .
\end{array}\right.
$$

And in the circular ring $4<|z|<5, \gamma(z)$ shall be equal to the solution of Dirichlet problem with the boundary values $g\left(z^{*}\right)$ on $|z|=4,0$ on $|z|=5$.

Let $\delta>0$ be a sufficiently small number, with which we define the function 


$$
\gamma_{0}(z)=\left\{\begin{array}{lr}
g(z) & |z|<1 \\
\gamma(z) & 1<|z| \leq 2 \\
M(\gamma ; z ; \delta(|z|-2)) & 2 \leq|z| \leq 3 \\
M(\gamma ; z ; \delta) & |z| \geq 3
\end{array}\right.
$$

We see first, $\gamma_{0}(z)$ is locally Hölder-continuous with exponent $\alpha$ outside of the unit circle, and then globally as well, there. If $\left|z_{1}\right|<1<$ $\left|z_{2}\right|<2,\left|\gamma_{0}\left(z_{1}\right)-\gamma_{0}\left(z_{2}\right)\right|=\left|\gamma_{0}\left(z_{1}\right)-\gamma_{0}\left(z_{2}^{*}\right)\right| \leq A\left|z_{1}-z_{2}^{*}\right|^{\alpha} \leq A\left|z_{1}-z_{2}\right|^{\alpha}$, since $|z|=1$ is Apollonius circle with respect to the pair $z_{2}, z_{2}^{*}$. So $\left|\gamma_{0}\left(z_{1}\right)-\gamma_{0}\left(z_{2}\right)\right| \leq$ const. $\left|z_{1}-z_{2}\right|^{\alpha}$ whenever $\left|z_{1}\right| \neq 1,\left|z_{2}\right| \neq 1$.

Put

$$
g_{n}(z)=M\left(\gamma_{0} ; z ; \delta / n\right)
$$

Then $\left\{g_{n}(z)\right\}$ is one of the desired sequences. Because, $\left\{g_{n}(z)\right\}$ possesses obviously the properties i), iii) and iv). As for ii), we have by definition

$$
\left|\gamma_{0}\left(z_{1}+r e^{i t}\right)-\gamma_{0}\left(z_{2}+r e^{i t}\right)\right| \leq B\left|z_{1}-z_{2}\right|^{\alpha}
$$

for almost all $r \in(0, \delta / n)$ and $t \in(0,2 \pi)$. Q.E.D.

Theorem 1. Let $\zeta=\varphi(z)$ be a continuously differentiable sensepreserving homeomorphism between the unit disks in $z\left(=x+i y=r e^{i t}\right)$ and $\zeta\left(=\xi+i \eta=\rho e^{i \theta}\right)$-plane respectively, which is conformal with respect to Riemannian metric $d s=|d z+h(z) d \bar{z}|$. Then, if $h(z)$ fulfils the Hölder condition of order $\alpha(0<\alpha \leq 1)$, the boundary function $\theta=\theta(t)$ is absolutely continuous.

Proof. We may assume $\varphi(0)=0, \varphi(1)=1$ without loss of generality. By Lemma we can choose a sequence of functions $\left\{h_{n}(z)\right\}$ converging uniformly to $h(z)$ in $|z|<1$, such that $\left|h_{n}\left(z_{1}\right)-h_{n}\left(z_{2}\right)\right| \leq B\left|z_{1}-z_{2}\right|^{\alpha}$ for any $z_{1}$ and $z_{2}, h_{n}(z)=0$ outside of some compact set, and that $\left|h_{n}(z)\right|$ $\leq k<1$. Moreover, we may assume that the sequence $\left\{h_{n}(z)\right\}$ is uniformly convergent for $|z|<\infty$, since it forms a normal family there on account of the condition ii). Now, it is possible to construct the unique mapping $Z=\varphi_{n}(z)$ which is conformal in the metric $d s=\mid d z+$ $h_{n}(z) d \bar{z} \mid$ and supplies a homeomorphism between the whole $z$ - and $Z$ plane with the normalization

$$
\varphi_{n}(0)=0, \quad \varphi_{n}(\infty)=\infty, \quad \lim _{z \rightarrow \infty} \frac{\varphi_{n}(z)}{z}=1
$$

(cf. [2]). Then the sequence $\left\{\varphi_{n}(z)\right\}$ converges to a quasi-conformal mapping, $\zeta=\varphi_{0}(z)$ say, from $|z| \leq \infty$ to $|\zeta| \leq \infty$ (cf. [1]). Further it was proved in Ahlfors [3] that $\varphi_{0}(z)$ is conformal in $d s=|d z+h(z) d \bar{z}|$ almost everywhere in $|z|<1$ as follows: Soppose that $\varphi_{0}(z)$ is totally differentiable at $z=z_{0}\left(\left|z_{0}\right|<1\right)$ and set

$$
d \varphi_{0}\left(z_{0}\right)=p\left(z_{0}\right) d z+q\left(z_{0}\right) d \bar{z} .
$$

A small square $Q$, with dimension $d$, centred at $z_{0}$ is, if fixed in a suitable direction, transformed by the locally affine mapping $\varphi_{n}$ to a curvilinear quadrilateral $\Delta_{n}$ which is situated very near the rectangle 
of module $\left(\left|p\left(z_{0}\right)\right|+\left|q\left(z_{0}\right)\right|\right) /\left(\left|p\left(z_{0}\right)\right|-\left|q\left(z_{0}\right)\right|\right)$, so far as $n$ is sufficiently large. One has

$$
\begin{aligned}
& \left|p\left(z_{0}\right)\right|+\left|q\left(z_{0}\right)\right| \\
& \left|p\left(z_{0}\right)\right|-\left|q\left(z_{0}\right)\right|
\end{aligned} \bmod \Delta_{n}+\varepsilon \leq \frac{1}{d^{2}} \iint \frac{1+\left|h_{n}(z)\right|}{1-\left|h_{n}(z)\right|} d x d y+\varepsilon
$$

by a slight modification of the module theorem. Let $n \rightarrow \infty$ and then $d \rightarrow 0$. Thus $|q(z) / p(z)| \leq|h(z)|$ almost everywhere in $|z|<1$. Changing the independent variable by a sense-preserving affine transformation $z=a_{\tau}+b_{\bar{\tau}}(|a|>|b|)$, one gets finally $q(z) / p(z)=h(z)$ a.e. in $|z|<1$.

Let $D$ be the image of the unit disk by $Z=\varphi_{0}(z)$. We map $D$ conformally onto the unit disk $|\zeta|<1$ by $\zeta=F(Z)$, so that $Z=0, \varphi_{0}(1)$ corresponds to $\zeta=0,1$, respectively. Then we see that for $|z|<1$

$$
\varphi(z)=F_{\circ} \varphi_{0}(z) \text {. }
$$

Let us denote $\psi_{n}(z)=\varphi_{0}(z)-z$. Then, for any rectifiable Jordan curve $C$, there holds the well-known Pompeiu's formula

$$
\psi_{n}(z)=\frac{1}{2 \pi i} \int_{C} \frac{\psi_{n}(w)}{w-z} d w+\frac{1}{\pi} \int_{[C]} \frac{q_{n}(w)}{z-w} d u d v \quad w=u+i v,
$$

where $[C]$ is the interior of $C$ and $q_{n}(z)=\partial \psi_{n}(z) / \partial \bar{z}$. This can be brought to the form

$$
\psi_{n}(z)=\frac{1}{\pi} \iint \frac{q_{n}(w)}{z-w} d u d v
$$

as $C \rightarrow \infty$, in virtue of the normalization. Since $q_{n}(z)$ is Hölder-continuous for $|z|<\infty$ (cf. [2]), we have

$$
\frac{d \psi_{n}\left(e^{i t}\right)}{d t}=-i\left[e^{-i t} q_{n}\left(e^{i t}\right)+\frac{e^{i t}}{\pi} \iint \frac{q_{n}(w)}{\left(e^{i t}-w\right)^{2}} d u d v\right],
$$

where the right-hand integral is to be taken as Cauchy principal value about $e^{i t}$. We can extract from $\left\{q_{n}(z)\right\}$ a suitable subsequence $\left\{q_{n_{\nu}}(z)\right\}$ uniformly convergent everywhere. Since

$$
\iint \frac{q_{n}(w)}{\left(w-e^{i t}\right)^{2}} d u d v=\iint \frac{q_{n}(w)-q_{n}\left(e^{i t}\right)}{\left(w-e^{i t}\right)^{2}} d u d v
$$

and

$$
\left|\frac{q_{n}(w)-q_{n}\left(e^{i t}\right)}{\left(w-e^{i t}\right)^{2}}\right| \leq m\left|w-e^{i t}\right|^{\beta-2} \quad(0<\beta<\alpha),
$$

there exists by Lebesgue's theorem

$$
\lim _{\nu \rightarrow \infty} \frac{d \psi_{n_{\nu}}\left(e^{i t}\right)}{d t}=-i\left[\frac{e^{i t}}{\pi} \lim _{\nu \rightarrow \infty} \iint \frac{q_{n_{\nu}}(w)}{\left(w-e^{i t}\right)^{2}} d u d v+e^{-i t} \lim _{\nu \rightarrow \infty} q_{n_{\nu}}\left(e^{i t}\right)\right],
$$

and $\left\{d \psi_{n_{\nu}}\left(e^{i t}\right) / d t\right\}$ is uniformly bounded for $0 \leq t \leq 2 \pi$. Therefore again by the same theorem

$$
\varphi_{0}\left(e^{i t_{0}}\right)-\varphi_{0}(1)-e^{i t_{0}}+1=\lim _{\nu \rightarrow \infty}\left[\psi_{n_{\nu}}\left(e^{i t_{0}}\right)-\psi_{n_{\nu}}(1)\right]=\int_{0}^{t_{0}}\left[\lim _{\nu \rightarrow \infty} \frac{d \psi_{n_{\nu}}\left(e^{i t}\right)}{d t}\right] d t,
$$

which implies that $\varphi_{0}\left(e^{i t}\right)$ is absolutely continuous function in $t \in(0,2 \pi)$. Thus the boundary $\Gamma$ of $D$ is rectifiable Jordan curve with the representation 


$$
Z=Z(t) \quad 0 \leq t \leq 2 \pi,
$$

and this function transforms any set of linear measure zero on $|z|=1$ to a set of linear measure zero on $\Gamma$. Simple application of Riesz's theorem yields the absolute continuity of the function

$$
\theta=\arg \varphi\left(e^{i t}\right)
$$

in view of $(*)$. Q.E.D.

By pseudo-analytic function in a domain $D$ in $z$-plane we imply the function $w=f(z)=u(x, y)+i v(x, y)$ satisfying there the conditions:

i) $f(z)$ is defined, one-valued and continuous;

ii) $u_{x}, u_{y}, v_{x}, v_{y}$ exist and continuous;

iii) $J(z)=u_{x} v_{y}-u_{y} v_{x}>0$ with possible exception of at most the countable set $S$ of points where $J(z)=0$, which accumulates nowhere inside of $D$;

iv) Dilatation of $f(z)$ is uniformly bounded for $z \notin S$.

It is evident that the function $h(z)=f_{\bar{z}} / f_{z}$ is defined and continuous for any point $z \notin S$. Here may be imposed on this eccentricity function $h(z)$ the further restriction $(\mathrm{H})$ :

(H) $\left\{\begin{array}{l}1) \text { for any point } z_{0} \in S, \lim _{z \rightarrow z_{0}} h(z) \text { exists; } \\ \text { 2) } h(z) \text { is Hölder-continuous with some exponent } \alpha \text { through- } \\ \text { out } D \text { after the continuous prolongation } 1)\end{array}\right.$

Then we have an extension of Fatou's theorem:

Theorem 2. Let $w=f(z)$ be a pseudo-analytic function in the unit disk $|z|<1$. If $f(z)$ is bounded and subject to the condition $(H)$ in its definition domain, $f(z)$ possesses the well-determined limit values as $z$ tends along Stolz paths to the periphery point $e^{i t}$ for every value of $t \in(0,2 \pi)$ except possibly for a set of linear measure zero.

Proof. Let $R$ be the Riemann configuration generated by $w=f(z)$. It can be considered as the map by the analytic function $w=F(\zeta)$ for $|\zeta|<1\left(F(0)=f(0), F^{\prime}(0)>0\right)$. Then $\frac{\partial \zeta}{\partial \bar{z}} / \frac{\partial \zeta}{\partial z}=h(z) . \quad F(\zeta)$ has an angular limit at every point of a set $E$ of measure $2 \pi$ on $|\zeta|=1$. By means of the quasi-conformal mapping $\zeta=\zeta(z), E$ corresponds to a set of measure $2 \pi$ on $|z|=1$ on account of Theorem 1, while any Stolz path in $|z|<1$ is transformed to another such in $|\zeta|<1$ (cf. [6]). It follows that $f(z)$ has the property asserted. Q.E.D.

Some other theorems concerning the boundary correspondence by conformal mappings or the boundary values of analytic functions can be generalized in similar manners.

\section{References}

[1] Ahlfors, L. V.,: On quasiconformal mappings, J, d'Anal, Math., 3, 1-58 (19531954). 
[2] - Conformality with respect to Riemannian metrics, Ann. Acad. Sci. Fenn. A. I., 206, 1-22 (1955).

[3] - On quasiconformal mappings, Lecture at Osaka University, February (1956)

[4] Beurling, A., and Ahlfors, L. V.,: The boundary correspondence under quasiconformal mappings, Acta Math., 96, 125-142 (1956).

[5] Lavrentieff, M.,: Sur une classe de représentations continues, C. R. Acad. Sci., Paris, 200, 1010-1012 (1935).

[6] Mori, A.,: On quasi-conformality and pseudo-analyticity, Trans. Amer. Math. Soc., 84, 56-77 (1957). 